BNL-73381-2005-CP

\title{
Simulations of High-Energy Electron Cooling
}

\author{
A.V. Fedotov, I. Ben-Zvi, Yu. Eidelman, \\ V.N. Litvinenko, N. Malitsky, et. al. \\ To be presented at Particle Accelerator Conference \\ Knoxville, Tennessee \\ May 16-20, 2005
}

Collider-Accelerator Department

Brookhaven National Laboratory

P.O. Box 5000

Upton, NY 11973-5000

www.bnl.gov

Managed by

Brookhaven Science Associates, LLC

for the United States Department of Energy under

Contract No. DE-AC02-98CH10886 


\section{DISCLAIMER}

This report was prepared as an account of work sponsored by an agency of the United States Government. Neither the United States Government nor any agency thereof, nor any of their employees, nor any of their contractors, subcontractors, or their employees, makes any warranty, express or implied, or assumes any legal liability or responsibility for the accuracy, completeness, or any third party's use or the results of such use of any information, apparatus, product, or process disclosed, or represents that its use would not infringe privately owned rights. Reference herein to any specific commercial product, process, or service by trade name, trademark, manufacturer, or otherwise, does not necessarily constitute or imply its endorsement, recommendation, or favoring by the United States Government or any agency thereof or its contractors or subcontractors. The views and opinions of authors expressed herein do not necessarily state or reflect those of the United States Government or any agency thereof. 


\title{
SIMULATIONS OF HIGH-ENERGY ELECTRON COOLING*
}

\author{
A.V. Fedotov ${ }^{\#}$, I. Ben-Zvi, Yu. Eidelman, V. N. Litvinenko, N. Malitsky, BNL, Upton, NY 11973 \\ I. Meshkov, A. Sidorin, A. Smirnov, G. Trubnikov, JINR, Dubna, Russia \\ D.L. Bruhwiler, Tech-X, Boulder, CO 80303
}

\section{Abstract}

High-energy electron cooling of RHIC presents many unique features and challenges. An accurate estimate of the cooling times requires a detailed calculation of the cooling process, which takes place simultaneously with various diffusive mechanisms in RHIC. In addition, many unexplored effects of high-energy cooling in a collider complicate the task of getting very accurate estimates of cooling times. To address these high-energy cooling issues, a detailed study of cooling dynamics based on computer codes is underway at Brookhaven National Laboratory. In this paper, we present an update on code development and its application to the high-energy cooling dynamics studies for RHIC.

\section{INTRODUCTION}

Existing electron cooling systems are based on an electron beam generated with electrostatic DC electron gun, immersed in a longitudinal magnetic field. For cooling of $100 \mathrm{GeV} / \mathrm{u}$ gold ions in RHIC, $55 \mathrm{MeV}$ electrons are needed. This makes the use of electrostatic acceleration unviable. RF acceleration of a bunched electron beam results in an electron transverse momentum spread which is orders of magnitude larger than in existing coolers. This large transverse temperature of the electron beam can be effectively used to suppress the recombination of $\mathrm{Au}$ ions [1]. For cooling purposes, the large temperature can be controlled with a strong magnetic field in the cooling solenoid, making magnetized cooling an attractive approach [1]. Another approach based on non-magnetized cooling is also under consideration [2].

An order of magnitude estimate of cooling time can be obtained using simple expressions for the cooling force. For example, using the non-magnetized expression for the force, we can express the inverse cooling time, for a given transverse beam emittance as:

$$
\frac{1}{\tau}=\frac{4 \pi Z^{2} r_{p} r_{e} n_{e} c \eta \Lambda_{c}}{A \gamma^{2}}\left[\frac{\beta_{i}}{\gamma \varepsilon_{i}}\right]^{3 / 2},
$$

where $\mathrm{A}$ and $\mathrm{Z}$ are the ion atomic and charge numbers, $\gamma$ and $c$ are relativistic factor and the speed of light, $\beta_{i}$ is the beta-function in the cooling section, $\eta$ is the length of the cooling region divided by the ring circumference, $\Lambda_{c}$ is the Coulomb logarithm, $r_{e}$ and $r_{p}$ are the classical electron and proton radii, $\mathrm{n}_{\mathrm{c}}$ is electron beam density in the laboratory frame, and $\varepsilon_{\mathrm{i}}$ is the normalized $\mathrm{rms}$ ion beam emittance.

For RHIC parameters [3], the cooling time of $\mathrm{Au}$ ions

\footnotetext{
*Work supported by the U.S. Department of Energy
}

"fedotov@bnl.gov at $100 \mathrm{GeV} / \mathrm{u}$ is of the order of $1000 \mathrm{sec}$, which is much longer than typical cooling time of about $0.1-1 \mathrm{sec}$ in standard low-energy coolers. As a result, order of magnitude accuracy in the cooling time prediction for RHIC is unacceptable. An accurate estimate of cooling times requires detailed calculations of the cooling process, which takes place simultaneously with various diffusive mechanisms in RHIC. Performance optimization requires stateof-the-art computer simulations, some of which are described in this paper.

\section{COOLING DYNAMICS SIMULATIONS}

\section{Cooling Force}

The first step towards accurate calculation of cooling times is to use an accurate description of the cooling force. The achievable Coulomb logarithm in the analytic expression for the cooling force is not very large for the RHIC electron cooler [3]. As a result, accuracy of the friction force formulas, derived in a logarithmic approximation, may be questionable. In addition, in some regimes there is a significant discrepancy between available formulas. For this reason the ParSEC project at Tech-X Corp. is developing parallel modeling capability, based on the VORPAL code [4], to simulate from first principles the friction force and diffusion coefficients for RHIC parameters [5].

Our goal is to explore the validity of available formulas for regimes with various degrees of magnetization, especially the one which is expected for cooling in RHIC. The goal is also to determine quantitatively the effect of complicated factors, such as bulk space-charge electric fields, variations of electron density, the effect of nearby trajectories of other ions, impact of errors in the magnetic field of cooling solenoid, etc. Significant efforts were made to establish an accurate simulation approach for the magnetized cooling [5]. The updated version of the code is presently being used to explore various aspects of the friction force for RHIC parameters [6]. In addition, simulations of the friction force and its dependence on various parameters is being verified vs recent measurements at CELSIUS [7].

\section{Cooling Dynamics Codes}

Two cooling dynamics codes are being developed for the RHIC project - SIMCOOL [8] and BETACOOL [9].

SIMCOOL was originally developed at BINP in Novosibirsk. It is presently being developed by the BNL team with on-going improvements being made to address specifics of electron cooling in RHIC. Some of the recent modifications to the code include synchrotron motion, more accurate treatment of the longitudinal and transverse 
diffusion due to IBS, and development of a core-tail model for the IBS treatment [10]. The modeling allows for a variable ion beam distribution as a result of both cooling and IBS, which leads to a beam distribution with a dense core. Formation of such a core is an important feature, since it plays a dominant role in the luminosity increase. In fact, even for parameters of the cooler, which may not be sufficient for reducing the rms emittance, a pronounced core can lead to a strong luminosity increase. In such a case, an accurate treatment of other effects, which may depend on such a dense core, becomes extremely important.

BETACOOL is being developed at JINR in Dubna [9] in collaboration with the BNL team. Recently, the development of many effects in BETACOOL was done in parallel with SIMCOOL. A detailed cross-verification of the two codes increased our confidence in the accuracy of the models.

Many modifications in the code were directly aimed at the specific questions of high-energy cooling at RHIC. Such developments include: 1) improved accuracy of the IBS calculation under cooling; 2) solenoid errors in the cooling section; 3 ) dynamically variable cooling parameters for luminosity optimization; 4) accurate treatment of particle losses due to collisions, finite acceptance, loss from the bucket and recombination; 5) various approaches for calculation of the beam-beam parameter and the luminosity of ion distributions while cooling; 6) a numerical model for calculation of the non-magnetized friction force; and 7) numerical calculation of the recombination rate for electron motion in an undulator field.

A critical item of code development includes crosscheck between various models and verification of the models based on experimental data. For example, several models of the IBS were extensively cross checked to understand their accuracy and the validity of approximations being made. The models were verified vs dedicated IBS experiments during Run-4 of RHIC for Au ions and during Run-5 for $\mathrm{Cu}$ ions. Good agreement of the models with the experimental data [11] significantly increased our confidence in the accuracy of the models being used in cooling simulations for RHIC.

The main purpose of cooling is to counteract diffusion within the ion beam (i.e. emittance growth), which may be caused by various effects. For accurate treatment, a dynamic simulation code should include both cooling and all diffusion sources, including IBS, beam-beam effects, machine nonlinearity, etc. An accurate simulation of beam-beam effects and cooling acting simultaneously are planned in the framework of the Unified Accelerator Libraries (UAL) [12]. BETACOOL was recently integrated with the UAL framework [13].

\section{Simulations}

Various possibilities of using electron cooling at RHIC are described elsewhere [2]. For example, direct cooling at the top energy can be considered as a baseline approach for RHIC-II. However, for the eRHIC [14] project, it is important that cooling is fast enough to allow reduction of rms beam parameters, especially the rms bunch length. In this case, pre-cooling at low energy becomes very attractive due to a very strong dependence of the cooling time on energy. For the same reason, cooling is very effective for scenarios with collisions at low energy.

Electron cooling of $\mathrm{Au}$ ions at the storage energy of 100 $\mathrm{GeV} / \mathrm{u}$ will bring into reach the desired increase of the integrated average luminosity for the RHIC upgrade, which is a factor of 40 larger than the original design value. For the RHIC-II upgrade, electron cooling will provide a minimum factor of 10 increase [2] in the luminosity of $\mathrm{Au}$ ions, as compared to the upgrade without cooling.

Figure 1 shows the luminosity for RHIC-II parameters (Table 1) based on the magnetized cooling approach [2]. For present RHIC operation without electron cooling, $\beta^{*}$ (the beta function at the IP) is limited to about one meter (or slightly less), because the emittance is increased a factor of two by IBS. Further reduction of $\beta^{*}$ with such an increase of emittance would lead to a significant angular spread and beam loss. On the other hand, keeping the rms emittance constant (by cooling), allows one to begin a store cycle with smaller values of $\beta^{*}$. This is taken into account in Fig. 1 , where $\beta^{*}=0.5$ and $1 \mathrm{~m}$ were used with and without electron cooling, respectively.

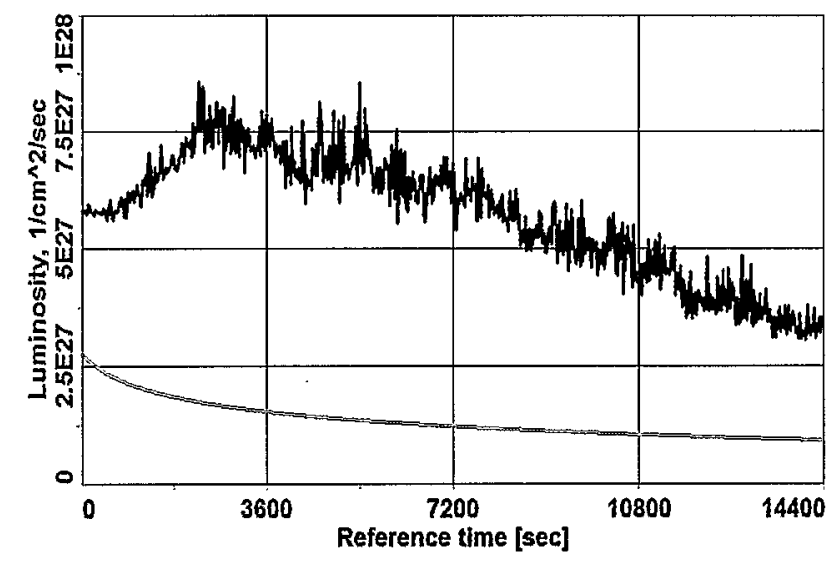

Figure 1: Simulated luminosity for the RHIC-II upgrade, with (blue top curve) and without (red) magnetized electron cooling, taking $\beta^{*}=0.5 \mathrm{~m}$ and $1 \mathrm{~m}$, respectively

The sharp initial increase in the luminosity during cooling, seen in Fig. 1, is associated with quick formation of a pronounced core in the beam distribution. For example, beam profiles after $1800 \mathrm{sec}$ of cooling are shown in Fig. 2. As a result, it becomes necessary to control ion beam-beam parameters. This is achieved by dynamically increasing the size of the electron beam, which allows one to keep the beam-beam parameter at the required level. For the luminosity shown in Fig.1, the beam-beam parameter (calculated based on the local density) was kept at 0.005-0.007 per IP, as is shown in Fig. 3, for the horizontal and vertical beam-beam parameters. 


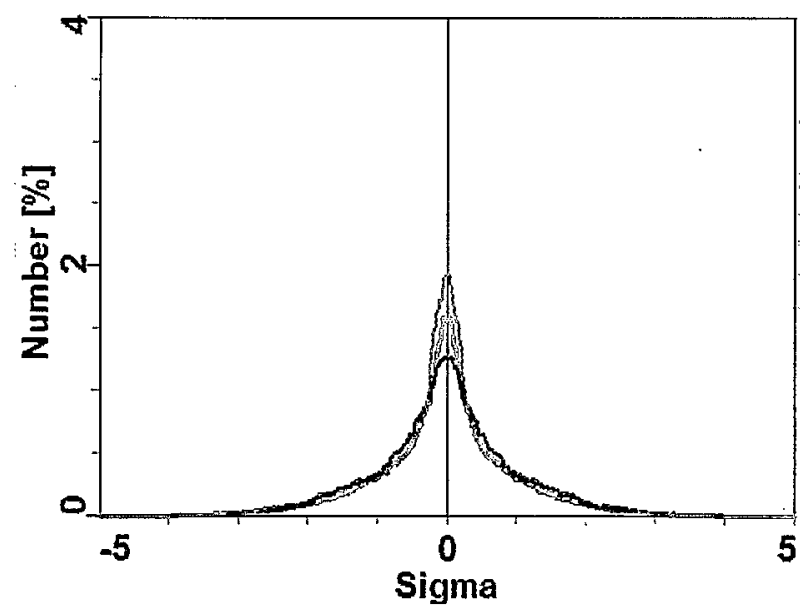

Figure 2: X (red), Y (blue) and longitudinal (green) beam profiles after $1 / 2$ hour of cooling.

Table 1: RHIC-II parameters for Au ions used in simulations

\begin{tabular}{|l|l|}
\hline Relativistic $\gamma$ & 108 \\
\hline Number of bunches & 112 \\
\hline Number of ions per bunch & $1^{*} 10^{9}$ \\
\hline Normalized emittance, $95 \%[\mathrm{~mm} \mathrm{mrad}]$ & $15 \pi$ \\
\hline
\end{tabular}

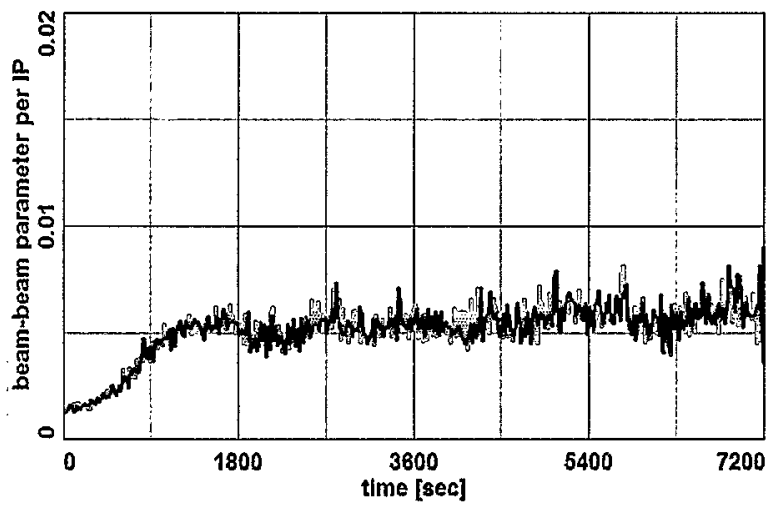

Figure 3: Time evolution of the beam-beam parameter ( $X$ $\& Y$ ), using dynamic optimization with the electron beam.

The slow decrease of the luminosity after its peak value (Fig.1, blue line) is due to the loss of Au ions at the IP. The cross section of such a "burn-off" process (from dissociation and bound electron-positron pair production) is about 212 barn, which limits the store time. To deliver the highest luminosity per store, the goal is to "burn" as much beam as possible. For the luminosity shown in Fig. 1 , about $60 \%$ of the beam is burned in 4 hours, as is shown in Fig. 4, which corresponds to an average luminosity of $7^{*} 10^{27}\left[\mathrm{~cm}^{-2} \mathrm{~s}^{-1}\right]$.

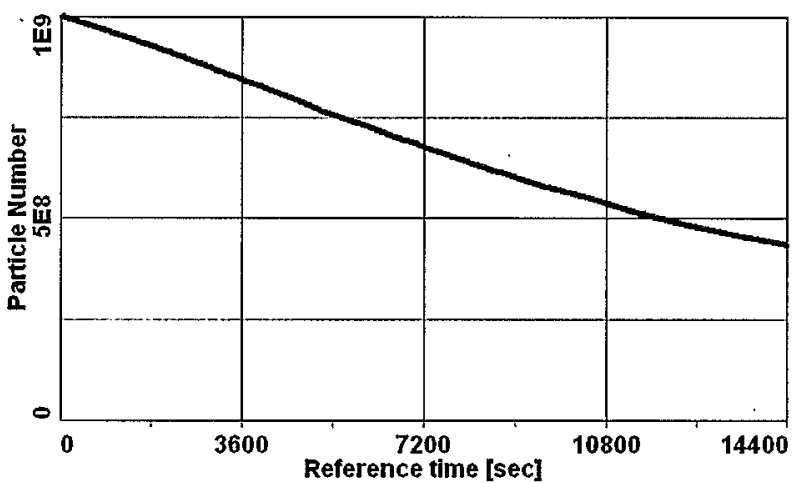

Figure 4: Bunch intensity decrease due to "burn-off" processes from $\mathrm{Au}-\mathrm{Au}$ collisions, assuming three IP's.

\section{ACKNOWLEDGMENTS}

We would like to thank D. Abell, A. Burov, Ya. Derbenev, W. Fischer, J. Kewisch, C. Montag, G. Parzen, T. Roser and the RHIC Accelerator Physics Group for many useful discussions during these studies. We also acknowledge collaboration on these topics with the INTAS project "Advanced beam dynamics in storage rings".

\section{REFERENCES}

[1] I. Ben-Zvi, V.V. Parkhomchuk, C-AD/AP/47 (2001).

[2] A.V. Fedotov et al., TPAT089, these proceedings.

[3] The RHIC E-cooler Design Report http://www.agsrhichome.bnl.gov/eCool

[4] C. Nieter, J. Cary, J. Comp. Phys. 196, p. 448 (2004).

[5] D. Bruhwiler et al., Proceedings of ICFA-HB2004 Workshop, Bensheim, Germany (2004).

[6] A.V. Fedotov et al., TPAT092, these proceedings.

[7] A. Fedotov, B. Galnander, V. Litvinenko, T. Lofnes, A. Sidorin, A. Smirnov, V. Ziemann, "Experiments towards high-energy cooling", in preparation.

[8] Yu. Eidelman, A.V. Fedotov "Recent developments of the SIMCOOL code", unpublished (2004).

[9] The BETACOOL program, http://lepta.jinr.ru

[10]A.V. Fedotov et al., TPAT091, these proceedings.

[11] J. Wei et al, Proceedings of ICFA-HB2004 Workshop, Bensheim, Germany (2004).

[12]N. Malitsky, R. Talman, "Unified Accelerator Libraries", AIP 391 (1996).

[13]N. Malitsky, A. Smirnov, G. Trubnikov et al., http://www.ual.bnl.gov (2004).

[14] The eRHIC Design Report http://www.agsrhichome.bnl.gov/eRHIC 